\title{
Measuring Consensus as the Midpoint of the Central Tendency
}

\author{
William T. Gavin and Geentanjali Pande
}

$I^{\prime}$ n November 2007, the Federal Open Market Committee (FOMC) announced several changes to the process that it uses to make and report economic projections. ${ }^{1}$ One thing that has not changed is that the Fed will continue to report two ranges of forecasts: (i) the full range, encompassed by the high and the low forecasts; and (ii) the central tendency range, encompassed by the high and the low of the group after the three highest and three lowest forecasts are deleted. The Fed does not release the individual forecasts or a single statistic, such as the mean or median, which are commonly used measures to summarize the consensus in surveys of individual forecasts.

A common method for determining the consensus of the FOMC is to take the midpoint of the central tendency range. The question is how this measure of consensus compares with more traditional statistics, such as the mean and the median.

We can gauge the reliability of the FOMC's central tendency as a summary of the consensus of individual forecasts by using the Survey of Professional Forecasters (SPF) as a proxy. The SPF is conducted four times per year by the Federal Reserve Bank of Philadelphia, which maintains a database of the results, including the individual forecasts.
Here, we construct a central tendency range for the SPF and then compare it with the mean and median of the SPF respondents. Because the SPF often includes more than 19 forecasts (the maximum number possible for the Fed policy group), we compute the central tendency by eliminating two outliers (one high and one low) for every six forecasts. For a group the size of the FOMC, this is comparable to eliminating the top and bottom three forecasts.

The chart displays the midpoint of the SPF's central tendency range of the February forecasts for output and inflation as well as the mean and median of these same forecasts. Here the output and inflation measures are those reported by the Bureau of Economic Analysis, which switched from GNP to GDP in 1992. The left panel shows that, over the past 25 years, the midpoint of the central tendency range for the SPF output forecasts is almost identical to the mean and median. The right panel displays similar results for the inflation forecasts. Therefore, we may conclude that the midpoint of the SPF central tendency is a good approximation of the conventional measures of consensus like mean and median.

${ }^{1}$ See "Can You Hear Me Now?" by Kevin L. Kliesen (Economic Synopses No. 1, 2008) for a description of these changes.

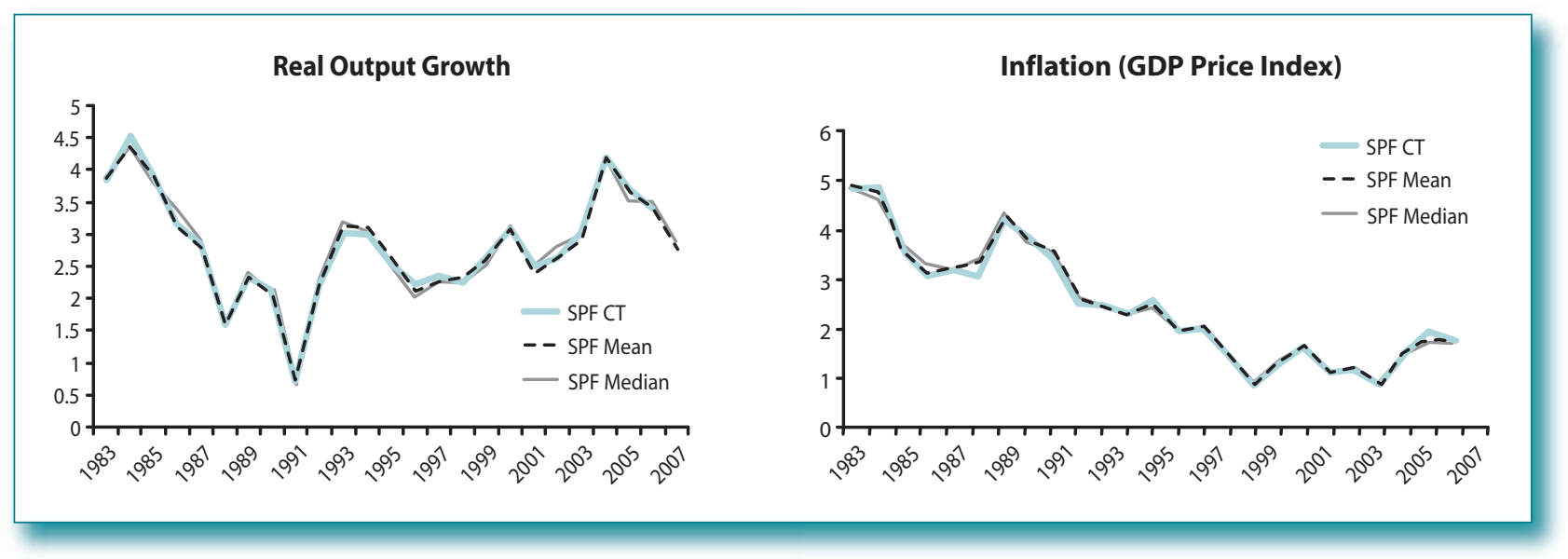

Views expressed do not necessarily reflect official positions of the Federal Reserve System. 